\title{
Theorie der Medizin
}

\section{J. Steurer}

Das «Horten Zentrum für praxisorientierte Forschung und Wissenstransfer» der Universität Zürich organisierte im September 2004 ein einwöchiges Seminar mit Professor Olli Miettinen von der McGill-Universität in Montreal. Das Seminar war eine Einführung in die Konzepte und Prinzipien - Theorie - der direkt praxisorientierten medizinischen Forschung.

Zu Beginn des Seminars gab Professor Miettinen eine kurze Einführung in seine neulich erschienenen Publikationen über die Theorie der Medizin. Diese Schriften standen den Teilnehmern des Seminars zur Verfügung und werden in diesem Artikel zusammengefasst. Die Zusammenfassung basiert zudem auch auf der persönlichen Kommunikation mit Miettinen. Mit diesem Artikel möchten wir in der Schweiz zu einer Diskussion über das Wesen und die Wissensbasis der Medizin anregen.

Ein Spruch von Francis Bacon (1561-1626), den Miettinen im Seminar wiederholt zitierte, stelle ich auch hier an den Anfang: «Read not to contradict, nor to believe, but to weigh and consider» [1].

\section{Wesen der Medizin}

Selbst wenn die Medizin idealerweise wissenschaftlich ist, ist die Medizin keine Wissenschaft, sondern ein Aggregat von Künsten (arts), bestehend aus den einzelnen Spezialitäten der Medizin [2]. Die Wissenschaften unterscheiden sich von den Künsten in der Natur ihrer Produkte. Die Produkte der Wissenschaften sind in ihrer Natur Episteme (Aristoteles), d.h. generelles ort- und zeitunabhängiges, abstraktes - Wissen, während die Produkte der Künste von Menschen geschaffene Dinge oder Handlungen sind. Diagnosen und Prognosen repräsentieren nicht generelles Wissen, deshalb ist deren Produktion Kunst, nicht Wissenschaft.

Hippokrates, der Vater der empirisch-rationalen Medizin, hat die medizinischen Begriffe der Diagnose und Prognose geschaffen, und die Dualität «Diagnose» und «Prognose» bestand bis vor kurzem. Erst kürzlich hat Miettinen eine zusätzliche Entität, die Ätiognose [3], eingeführt. Diagnose, Ätiognose und Prognose werden von Miettinen mit einem einzigen Wort als Gnose (gnosis) bezeichnet [3]. Diagnose ist aber nicht die Krankheit des Patienten; Ätiognose ist nicht deren kausaler Ursprung, Ätiogenese [4]; und Prognose ist nicht der zukünftige Verlauf der Gesundheit.

Gnose ist eine besondere Gattung von Wissen. Es ist das Wissen des Arztes über die Gesundheit des Klienten (Patient, gesunde Person oder Population). Gnose ist hiermit die wesentliche Voraussetzung, damit der Arzt für den Klienten nützlich sein kann. Gnose kann aber nicht sicheres Wissen über die Gesundheit des Patienten sein. Es ist probabilistisches Wissen, und die Theorie der Medizin definiert, im Prinzip, die korrekten Wahrscheinlichkeiten [5].

Die Diagnose in der klinischen Medizin ist das Wissen darüber, ob bei einem Individuum eine besondere Krankheit vorliegt oder nicht. Der Klient ist charakterisiert durch ein «diagnostisches Profil», ein Set verfügbarer und relevanter Fakten. Das diagnostische Profil basiert auf diagnostischen Indikatoren. Diese sind einerseits Risikoindikatoren und andererseits beschreiben die Indikatoren Symptome, Befunde bei der körperlichen Untersuchung und eventuell Resultate von Laboruntersuchungen. Ein einfaches Beispiel für ein diagnostisches Profil: 40jähriger Nichtraucher, mit Husten seit drei Tagen, kein Auswurf und Fieber von $39,5^{\circ} \mathrm{C}$.

Bei einem Patienten mit diesem diagnostischen Profil stellt sich zuerst die Frage, welche Krankheit mit diesem Profil vereinbar ist. In diesem Fall kann es eine Pneumonie sein, und nun stellt sich die nächste Frage, was ist die korrekte Wahrscheinlichkeit, dass der Patient an einer Pneumonie erkrankt ist. Diese Wahrscheinlichkeit ist unabhängig davon, ob er von einem Hausarzt oder einem Facharzt für Pneumologie an einem Universitätsspital betreut wird. Die Herausforderung für den Arzt liegt darin, die korrekte Wahrscheinlichkeit, dass es sich um eine Pneumonie handelt, zu kennen.

In dieser Situation entspricht die korrekte diagnostische Wahrscheinlichkeit einer Pneumonie, auch wenn man die Wahrscheinlichkeit nicht genau kennt, dem Anteil von Fällen mit diesem Profil, im generellen, bei dem eine Pneumonie vorhanden ist. Mit anderen Worten: Die korrekte Wahrscheinlichkeit entspricht der Prävalenz einer Pneumonie in Fällen wie diesen (Profil) im generellen [6]. 
Ein Beispiel für Prognose: Die korrekte prognostische Wahrscheinlichkeit entspricht dem proportionalen Anteil von Fällen mit einer Pneumonie mit einem besonderen prognostischen Profil, bei dem die gewählte Therapie zu einer Heilung führt.

\section{Wissensbasis der Medizin}

Vom Wesen der korrekten gnostischen Wahrscheinlichkeiten folgt, dass das gnostische Wissen im Prinzip auf dem basiert, was der Arzt über die relevante generelle Grösse weiss. Diese gnosisrelevante Grösse ist für alle drei Typen der Gnose eine profilspezifische Proportion. Der vom Arzt angenommene Wert der relevanten Proportion ist der Wert der entsprechenden gnostischen Wahrscheinlichkeit.

Das generelle Wissen, das relevant für Gnose ist, ist die Wissensbasis (knowledge base) der Medizin. Dieses generelle Wissen wird in der Praxis auf den einzelnen Klienten bezogen, und das Resultat ist Ad-hoc-(partikularistisches) Wissen. Während das generelle Wissen orts- und zeitungebunden ist, bezieht sich das resultierende Ad-hoc-Wissen auf einen einzelnen Klienten zu einem bestimmten Zeitpunkt. In diesem Sinn ist Gnose die Schnittstelle zwischen der medizinischen Praxis und dessen Wissensbasis.

Miettinen definiert die wissenschaftliche Medizin auf der Basis eines rationalen Gedankengerüsts (wie oben erwähnt) und die Anwendung von wissenschaftlichem Wissen im Rahmen dieses «Frameworks» [7].

Die Grundlage der wissenschaftlichen Wissensbasis der Medizin ist Forschung, die zum Ziel hat, die Wissensbasis für die Gnose zu bilden. Einzelne Studien liefern die Evidenz, und ausgehend von dieser wird von der «wissenschaftlichen Gemeinschaft» (scientific community) für das entsprechende Gebiet die Wissensbasis formuliert [2].

Dieses Wissen hat die Form von gnostischen Probabilitätsfunktionen. Zum Beispiel: Wissen, das für die Diagnose einer Pneumonie relevant ist, umfasst Wissen, das die Prävalenz der Pneumonie in Abhängigkeit von Husten, Höhe des Fiebers usw. beschreibt. Wenn die relevante Funktion, die diese Abhängigkeit beschreibt, im Computer des Arztes gespeichert ist, so kann dieser das jeweilige gnostische Profil in den Computer eingeben und der Computer berechnet die korrespondierende wissenschaftlich-gnostische Probabilität [8].

Da es noch eine gute Weile dauern wird, bis ein solche wissenschaftliche Wissensbasis auf breiter Ebene existiert und für praktizierende Ärzte zugänglich und brauchbar ist, sollte in der Zwischenzeit die entsprechende quasi-wissenschaftliche Wissensbasis der Praxis entwickelt werden [8]. Das Wissen darüber, was die korrekten gnostischen Wahrscheinlichkeiten im Rahmen verschiedener gnostischer Belange und Profile betrifft, kann von Expertenansichten hergeleitet werden. Basierend auf diesen Daten kann die Wissensbasis der quasi-wissenschaftlichen Medizin, wie bei der wissenschaftlichen Wissensbasis, in der Form von gnostischen Probabilitätsfunktionen beschrieben werden [8].

So lange aber das quasi-wissenschaftliche Wissen nicht verfügbar ist, bleibt es immer noch wichtig, sich bewusst zu sein, was die korrekten gnostischen Wahrscheinlichkeiten im Prinzip sind.

Ausgehend von den gnostischen Wahrscheinlichkeiten, die dem Wissen über die vermuteten Proportionen entsprechen, unterrichtet der Doktor das Individuum über seine Gesundheit [9]. Der Doktor unterrichtet den Klienten über seinen Gesundheitszustand (per Diagnose), möglicherweise über die Ätiologie/Ätiogenese [4] der Krankheit (per Ätiognose) und über den zukünftigen Verlauf seiner Gesundheit (per Prognose), inklusive der Effekte von Interventionen auf den zukünftigen Verlauf der Gesundheit.

Das Wissen über gnostische Probabilitäten, das zu den Ärzten (ihren Computern) transferiert wird, kann als gnostische Guideline beschrieben werden. Während Guidelines dieser Art sehr erstrebenswert sind, trifft dies auf die heute produzierten, vorwiegend entscheidungsorientierten Guidelines nicht zu [10]. Entscheidungen über Handlungen, Untersuchungen oder Interventionen sollten, wenn möglich, dem Klienten überlassen werden [11].

\section{Zum Schluss}

Als Folge dieser theoretischen Überlegungen formuliert Miettinen die fundamentale These, dass eine wissenschaftliche Medizin nur in dem Mass möglich ist, in dem die wissenschaftlichen Experten die konkrete Bedeutung der wissenschaftlichen Medizin in Form von gnostischen Guidelines definiert haben. Aber er fügt hinzu, dass es noch lange dauern wird, bis eine wissenschaftliche Wissensbasis auf breiter Ebene existiert. Das entsprechende Wissen für die quasi-wissenschaftliche Medizin könnte indes ziemlich rasch geschaffen werden. Es würde mich freuen, wenn diese Zusammenfassung von Miettinens Schriften über die Theorie der Medizin eine Diskussion 
zu diesem neuen Thema initiierte. Das Thema ist wichtig: Medizinische Gnosis bezieht sich üblicherweise auf Handeln [9] und Miettinen zitiert in diesem Zusammenhang [5] Ludwig von Mises, den bedeutenden Theoretiker menschlichen Handelns: «Action without thinking, practice without theory are unimaginable»[12].

\section{Literatur}

1 Bacon F. Essays 1597. In: Vickers B (ed.). Francis Bacon. The essays or counsels civil and moral. New York: Oxford University Press; 1999. p. 134.

2 Miettinen OS. The modern scientific physician: 1. Can practice be science? CMAJ 2001;165(4): 441-2.

3 Miettinen OS. Evidence in medicine: invited commentary [editorial]. CMAJ 1998;158(2): 215-21.

4 Miettinen OS, Flegel KM. Elementary concepts of medicine: VI. Genesis of illness: pathogenesis, aetiogenesis. J Eval Clin Pract 2003;9(3):325-7.
5 Miettinen OS. The modern scientific physician: 7. Theory of medicine. CMAJ 2001;165(10): 1327-8.

6 Miettinen OS. The modern scientific physician: 3. Scientific diagnosis. CMAJ 2001;165(6):781-2

7 Miettinen OS. The modern scientific physician: 2. Medical science versus scientific medicine. CMAJ 2001;165(5):591-2.

8 Miettinen OS. Evidence-based Medicine, Casebased Medicine; scientific medicine, quasi-scientific medicine. J Eval Clin Pract 2005;11: in press.

9 Miettinen OS, Flegel KM. Elementary concepts of medicine: IX. Acting on gnosis: doctoring, intervening. J Eval Clin Pract 2003;9(3):337-9.

10 Miettinen OS. The modern scientific physician: 8. Educational preparation. CMAJ 2001;165(11): 1501-3.

11 Miettinen OS, Flegel KM. Professionalism in medicine. J Eval Clin Pract 2003;9(3):353-6.

12 von Mises L. Human action. A treatise on economics. $3^{\text {rd }}$ edition. Chicago: Contemporary Books; 1966. p. 177. 